\title{
Strates
}

STRATES Matériaux pour la recherche en sciences sociales

14 | 2008

Espaces du quotidien

\section{Des mondes à soi. Pour une ethnologie des territoires du quotidien en HLM}

Denis La Mache

\section{OpenEdition}

1 Journals

Édition électronique

URL : http://journals.openedition.org/strates/6657

DOI : 10.4000/strates.6657

ISSN : $1777-5442$

Éditeur

Laboratoire Ladyss

Édition imprimée

Date de publication : 1 janvier 2008

Pagination : 55-68

ISBN : 0768-8067

ISSN : 0768-8067

Référence électronique

Denis La Mache, "Des mondes à soi. Pour une ethnologie des territoires du quotidien en HLM »,

Strates [En ligne], 14 | 2008, mis en ligne le 13 mars 2013, consulté le 08 septembre 2020. URL : http:// journals.openedition.org/strates/6657 ; DOI : https://doi.org/10.4000/strates.6657

Ce document a été généré automatiquement le 8 septembre 2020

Tous droits réservés 


\title{
Des mondes à soi. Pour une ethnologie des territoires $\mathrm{du}$ quotidien en HLM
}

\author{
Denis La Mache
}

1 Chaque matin, Madame T prend l'ascenseur, son caniche dans les bras. Elle descend treize étages, traverse le hall de l'immeuble avant qu'il ne soit investi par les «bandes de jeunes». C'est pour cela qu'elle a choisi de descendre tôt. Ce n'est pas qu'ils lui fassent peur, mais "comme on dit, on sait jamais ». Madame T relève sa boîte aux lettres puis remonte. En fin de matinée, quelques jeunes se rassemblent dans le hall. Parmi eux, Miloud E « passe le temps » en attendant d'aller « dans Tours ». Monsieur M, locataire du troisième, traverse le quartier, insensible à l'effervescence alentour. Il se rend à pied dans le centre de Saint-Pierre où, comme chaque jour, il « fait son tour ». Il ne semble pas ému par la présence des jeunes. Ce n'est qu'une apparence. Il me le confirmera lorsque, avec sa collaboration, j'explorerai les ressorts de son quotidien. Le plaisir de l'ethnologue est de mettre son nez chez les autres.

2 Nous sommes au début des années 2000, dans le grand ensemble de la Rabaterie. À Saint-Pierre-des-Corps, dans la banlieue de Tours, ce quartier HLM déploie 40 hectares d'urbanisme planifié. Au total, 2590 logements standardisés s'insèrent entre parkings et espaces verts. Quelques pavillons égarés rompent la régularité architecturale caractéristique d'une conception désormais révolue du logement social. Une population modeste et cosmopolite cohabite dans ce quartier classé Zone urbaine sensible, parfois soulevé par quelques « émeutes $^{1}$ ». Dans ce grand ensemble, une multitude de destins contrastés se juxtaposent et se succèdent depuis trente-cinq ans. Plus ou moins étranges ou étrangers les uns aux autres, des retraités, des ouvriers des jeunes ménages en transit vers le rêve pavillonnaire... partagent les lieux. Des quotidiennetés affairées composent les unes avec les autres. Il y a quelques années, j'avais exploré les logiques qui constituent l'art d'habiter que chacun déploie pour inscrire son existence dans ces conditions de logement ${ }^{2}$. Depuis, les matériaux d'enquête avaient été remisés, sans peut-être avoir livré tous leurs secrets. Une exploitation secondaire de ces fonds en 
terme de territoire du quotidien pourrait-elle encore produire du sens? L'hypothèse est stimulante mais le pari osé.

3 Entendue comme une répétitivité d'action dans une unité de temps convenue, la notion de quotidien est familière à l'observation ethnologique. Mais il s'agit ici de l'articuler à la notion de territoire. Ceci constitue à la fois l'intérêt et la difficulté de l'exercice. Vocable complexe et polysémique, le territoire résonne dans le langage des urbanistes, des politiques, plus rarement dans celui du citoyen. Coutumière de l'observation des relations des hommes à l'espace, l'ethnologie en fait d'ailleurs un usage prudent comme le rappellent Pierre Alphandéry et Martine Bergues (Alphandéry et Bergues, 2004). Dans une société marquée par l'augmentation des mobilités quotidiennes et résidentielles, les comportements évoluent et brouillent les cartes des ancrages. Il semble de plus en plus difficile de repérer des territoires entendus comme un ensemble de lieux collectivement approprié et délimité par une frontière reconnue et matérialisée par une histoire. Il existe, dit P. Alphandéry, de moins en moins de lieux auxquels un groupe social puisse s'identifier dans sa totalité (Alphandéry, 2001).

Convoquer la notion de territoire semble particulièrement une gageure dans un grand ensemble habité par une population hétérogène qu'aucun sentiment d'appartenance, aucune histoire, aucun consensus sur les usages de l'espace ne rassemble. Retorse à toute idée de culture locale, la vie sociale du quartier est caractérisée par l'hétérogénéité des pratiques spatiales de ses occupants. Le territoire du quotidien peut alors être envisagé, dans la perspective d'une anthropologie des singularités subjectives comme un espace dont chaque individu se donne la liberté de disposer chaque jour selon un usage singulier, un espace qui relève d'un modèle de découpage et de contrôle propre à l'individu considéré et pourvu d'un champ symbolique spécifique, garant d'identité.

5 Il semble possible de repérer des inventions contextuelles, des arts de faire par lesquels, dans la répétitivité de leurs pratiques quotidiennes, les habitants délimitent, administrent, transforment matériellement et symboliquement des espaces et des lieux. Nous entendrons par lieu une configuration instantanée de positions. L'idée de lieu, dit Michel de Certeau, exprime un ordre selon lequel des éléments sont distribués dans des rapports de coexistence. Les éléments y sont chacun à un endroit propre et distinct (Certeau, 1980). Il y a dans le lieu une indication de stabilité qui distingue ce dernier de l'espace défini par M. de Certeau comme intégrant les vecteurs de direction et de vitesse. L'espace est animé par ceux qui le pratiquent. Il est au lieu, dit M. de Certeau, «ce que devient le mot quand il est parlé ». Il y a, dit Maurice Merleau-Ponty, « autant d'espaces que d'expériences spatiales». Pour chacun, dit Michel Bonetti, l'espace suscite, recueille et condense "des images, des sentiments, des valeurs et des significations ». Fruit d'un bricolage, imaginaire, il est avant tout espace social (Bonetti, 1994). Il s'agit donc de voir comment les habitants transforment et relient ces espaces et ces lieux, en domptent les temporalités propres pour contribuer à faire territoire ${ }^{3}$.

Il est possible de postuler que l'observation des pratiques quotidiennes permet d'isoler des blocs d'espace-temps particuliers que chacun construit à sa mesure pour constituer son territoire du quotidien. L'existence de ces territoires est ici posée comme hypothèse heuristique, comme outil de compréhension permettant de saisir un ensemble d'actes dispersés. Il ne s'agit pas de supposer l'existence de territoires identifiés et cartographiables. Il s'agit de saisir de manière unifiée un registre particulier de fabrication sociospatiale sans rattacher les actes observés à une réalité 
strictement spatiale (un ensemble d'espaces et de lieux) ou sociale (un type de pratique).

7 Pour explorer la question, six familles seront évoquées ici. Les personnages cités ne sont pas des synthèses abstractives. Ils sont des individus bien réels issus d'une sélection méthodique. Au début de l'enquête, 760 foyers choisis aléatoirement sont venus constituer l'échantillon scruté par questionnaires. L'observation s'est ensuite resserrée sur un nombre plus réduit d'individus. Ils ne furent plus qu'une vingtaine de foyers soumis aux entretiens et à l'observation participante. Pour construire le propos présenté ici, l'effectif s'est encore réduit. Les autres sont là, en arrière-plan. Tapis dans les archives de l'enquête, ils laissent les faveurs du récit au petit groupe de ceux qui, sur les aspects abordés, présentent des caractères exemplaires.

\section{Fabriquer les territoires du quotidien}

8 Entre le «train-train » et l'exceptionnel, le « parfois » et le « souvent »... les habitants du quartier classent leurs actes en fonction de leur répétitivité. Ainsi apparaissent les pratiques quotidiennes, reconnues par leurs acteurs comme susceptibles de se reproduire chaque jour. Elles se donnent à voir dans une dimension spatiale et temporelle. Parfois simple cadre de réalisation, les espaces et les temps de ces pratiques quotidiennes, peuvent aussi en constituer une dimension nécessaire. Ils donnent alors lieu à des formes particulières de situations sociospatiales, c'est-à-dire de situations sociales dont l'espace est une dimension intrinsèque, à la fois cadre de réalisation et partenaire symbolique. C'est ce type de situation sociospatiale qui concentrera l'attention ici. Il s'agira d'explorer l'hypothèse selon laquelle les pratiques quotidiennes peuvent entretenir des relations de construction mutuelle avec des blocs d'espacetemps qui leur sont spécifiques. Il semble possible de proposer une exploration de la manière dont les individus fabriquent et transforment ces blocs d'espace-temps, bricolent dans les contraintes spatio-temporelles pour faire territoire du quotidien.

\section{Mobiliser des capitaux spécifiques pour réagir aux contraintes}

9 Les époux Croizer ont acheté un appartement dans un immeuble en accession à la propriété. Lui a 36 ans. Il est agent de service au collège du quartier. Elle a 33 ans. Elle est en congé parental et se consacre à l'éducation de leur fils âgé de 1 an au début de l'enquête. Quittant un habitat de centre-ville, ils se sont installés là "pour raison professionnelle ». La description de leurs pratiques quotidiennes fait apparaître des trajets, des « raccourcis », des " coins » bien à eux. S'ils forment un ensemble cohérent, les lieux de la pratique ne sont pas présentés comme une entité homogène. Ils se donnent à voir comme un ensemble éclaté. Comme l'avait remarqué Jean Remy, les individus ne vivent plus au centre d'un territoire monocentré et bien délimité (Remy, 1996). Celui de la famille Croizer est polycentrique et discontinu. Le jeune foyer vit dans plusieurs espaces et plusieurs temps impliquant une séparation de leurs activités. La structure de l'agglomération urbaine les amène à réagir à l'éclatement technique et social de l'espace en lieux spécialisés. Comme l'avait décrit Yannick Sencébé, ils évoluent dans une dissociation sociale des espaces de vie et se livrent à une multilocalisation des pratiques quotidiennes (Sencébé, 2003). Pourtant, l'individualisation des rapports à l'espace exprimée dans le quotidien ne constitue pas 
une explosion sociale et territoriale sans limites. Pour la famille Croizer, elle se partage essentiellement entre trois types de lieux: celui de l'emploi du mari, celui des commerces et des services et celui de la résidence. La multilocalisation des pratiques quotidiennes engendre des cheminements entre plusieurs lieux et milieux sociaux. Outre une importante pression temporelle, elle implique des contraintes multiples visà-vis desquelles la famille Croizer doit mobiliser ses capitaux sociaux, culturels et économiques. L'observation de la manière dont Mme Croizer « fait les courses » donne quelques enseignements.

10 L'activité professionnelle de son mari le soumet à des horaires contraints. Elle est " plus libre de [son] temps » et valorise cela comme un outil dans l'accessibilité urbaine. C'est donc le plus souvent seule que la jeune femme investit l'ensemble de l'agglomération. Son mode d'achat privilégié reste la fréquentation des hypermarchés de périphérie. L'usage de la voiture place dans le champ de ses possibilités objectives l'investissement de ce type de commerce. Mme Croizer a «les moyens d'aller partout». Dispositions techniques, temporelles et financières, les moyens résonnent dans les champs économiques et sociaux. Parfois, elle se rend dans les hard discounts. On y trouve dit-elle " des produits pas trop mal et pas chers ». Ce n'est pas sur le registre de la valorisation d'un faible pouvoir d'achat que Mme Croizer évoque ces enseignes. C'est sur celui de la satisfaction d'avoir "gagné de l'argent ». Mme Croizer aime remporter au nom de son foyer des victoires mêmes infimes sur un anonyme grand Capital. "J'ai été élevée comme ça [...] Chez moi, c'est la femme qui gère ce qui concerne la maison.» Elle remplie avec satisfaction cette part qu'elle s'assigne de son rôle familial. Régulièrement, elle aime aller "dans Tours faire les boutiques ». "C'est à un quart d'heure quand on passe par les quais à la bonne heure. Sinon, ça bouchonne jusqu'au boulevard Heurteloup. Le problème, c'est de se garer. Moi, je mets souvent la voiture vers la Loire. Faut marcher un peu mais c'est gratuit. » Pour «aller dans Tours ». Elle mobilise des trucs et des ruses qui lui facilitent la pratique de l'espace et en révèlent une grande connaissance. À Tours, Mme Croizer flâne et s'écarte des chemins convenus. La jeune femme connaît bien cette ville qu'elle a habitée dès l'âge de 11 ans, d'abord dans la maison de ses parents, puis seule dans un studio. Depuis, elle entretient une connaissance de la cité qu'elle veut riche et précise. Bien que désormais «en banlieue pour raison professionnelle", elle suit de près la valse des enseignes et l'évolution de la ville.

11 Mme Croizer aime présenter sa situation résidentielle actuelle comme " pas si éloignée de tout ». En évoquant ses habitudes de consommation, elle présente l'espace urbain comme un tissu nodal complexe et élargi de lieux entre les mailles duquel s'étale un désert sémantique appréhendé comme du temps de parcours qu'elle a su dompter. Ces lieux pratiqués lui sont plus que de pures topicités. Ils agissent comme des opérateurs actifs, structurés par des rythmes propres, dotés d'une histoire. La construction et l'entretien de cet ensemble spatio-temporel complexe trouvent leur justification dans la mobilisation active et inventive d'un système de positions et de possessions sociales, économiques et culturelles. Celles-ci s'expriment dans l'évocation de la culture familiale, du statut professionnel, de la stratégie résidentielle et de l'organisation du foyer. Les temporalités sont également au cœur de la construction des territoires du quotidien. Pour construire le sens de cet ensemble de lieux fréquentés, trois temps sont manipulés par Mme Croizer : le temps historique de la mémoire de la ville, le temps de la vie, des parcours sociaux et résidentiels et le temps quotidien des pratiques urbaines. 


\section{Produire de la distinction}

12 signe de distinction. Cette époque est bien révolue. L'arrivée dans le quartier constitue désormais une mise en danger de l'image de soi. Elle résulte de cheminements biographiques où se mêlent contraintes et adaptation, choix et résignation. Dans ce contexte, la multilocalisation des pratiques quotidiennes relie plusieurs lieux et liens sociaux. Elle permet d'entretenir, (plus rarement d'élargir) le champ des possibles en matière de réseaux de sociabilité. Les liens personnels que les individus tissent entre les contingences géographiques (accessibilité, distribution spatiale des lieux...) et sociales (réseaux de sociabilités accessibles...) pour construire les blocs d'espacetemps quotidiens servent des discours sur soi à l'adresse de soi-même et d'autrui. Dans un contexte général ou, comme le montrait J. Remy, la mobilité est socialement valorisée (Remy, 1996), les territoires du quotidien entretiennent avec les appartenances sociales et culturelles des relations intimes de construction et de renforcement mutuels. Faire territoire du quotidien sert des combats contre l'assignation.

Dans les étages supérieurs des tours, les époux Choquet entretiennent avec soin un appartement qui contraste avec les logements voisins par son dépouillement. "C'est que nous ne sommes pas là pour longtemps » explique Mme Choquet. Elle est sans emploi. Lui est ouvrier à l'entreprise Primagaz toute proche. Arrivés un an avant le début de l'enquête, la jeune femme de 24 ans et son mari de 26 ans sont là "pour débuter ». Le stigmate et la déqualification qui pèsent sur la cité irriguent la vision que le jeune ménage entretient du quartier. Leurs perspectives résidentielles espérées les leur rendent provisoires et donc plus supportables. Ils en supposent un poids plus important sur ceux qui sont condamnés à rester. Vis-à-vis des "gens d'ici», ils manifestent un respect compatissant. «C'est pas toujours drôle d'imaginer qu'on va passer toute sa vie dans un environnement pareil » souligne $\mathrm{M}$. Choquet. Le repli sur l'intérieur du logement leur paraît un moyen efficace de se soustraire de l'extérieur immédiat. Il permet d'échapper à la pression d'une forme dévalorisée d'habitat collectif. La mise à distance symbolique des "gens d'ici» s'accompagne d'un désinvestissement des parties communes. "À part mon pain le matin, raconte Mme Choquet, je reste tout le temps chez moi. Je suis toujours enfermée, sauf quand je fais les courses à Tours. Mais c'est tout. Je connais même pas Saint-Pierre presque [...] alors la cité... » Seules les composantes utilitaires sont fréquentées par défaut. "Quand je vois que tout est cassé, dégradé, que les gens font n'importe quoi...» Le quartier n'apparaît dans leur discours que comme du temps de parcours jusqu'à l'arrêt de bus, le centre commercial, le lieu de travail ou les domiciles de leurs amis. Il est un parcours ponctué de spectacles d'altérité. La Rabaterie n'est pas leur quartier. Ils se défendent d'en pratiquer une quelconque appropriation. Il est un «environnement » dont ils réduisent la fréquentation au minimum. Il est un espace de transit comme il est un lieu d'habitation transitoire.

Les territoires du quotidien se construisent avec des pleins, ici constitués d'espaces privés (les appartements) et d'espaces publics (Tours). Ils se construisent aussi avec des creux (le quartier) qui, dans ce cas, prennent sens au regard du système de contraintes qui régissent l'accès au logement. Comme l'avaient remarqué Jean-Claude Chamboredon et Madeleine Lemaire, l'attitude face au grand ensemble est fonction des chances de le quitter. Il est corrélé au degré de liberté face aux contraintes qui 
définissent les conditions de logement (Chamboredon, Lemaire, 1970). Stratégies d'investissement ou de désinvestissement d'espaces et de lieux par les pratiques quotidiennes, les territoires du quotidien se construisent de manière cohérente avec une stratégie sociale et résidentielle dont ils assurent la visibilité.

\section{Braconner dans des découpages institués}

15 Les habitants du quartier construisent ces blocs d'espace-temps qui forment les territoires du quotidien en utilisant la ville et ses recoins qu'ils découpent et assemblent. Ces découpages singuliers ne sont pas indépendants des configurations instituées. Les territoires du quotidien sont aussi des braconnages dans les territoires institutionnels.

16 M. Chabot a 35 ans. Sa femme en a 32. Il est ouvrier à la Sernam. Elle travaille à Tours comme secrétaire à temps partiel. Ensemble, ils élèvent leur fils âgé de 3 ans à la fin de l'enquête. Le jeune foyer se sent "plutôt bien » dans ce logement qui ne serait «pas si mal » s'il n'était « entouré de voyous ». Excentré par rapport au quartier, le Bloc des Jeux d'Eau offre tous les signes d'un « immeuble bien », hélas «trop proche de la ZUP ». Pour eux, en effet, "le bloc ne fait pas partie du grand ensemble». Tout dans leurs pratiques quotidiennes contribue à mettre à distance "la ZUP", si proche dans l'espace, si loin dans le style de vie revendiqué. L'exercice de mise à distance symbolique de «la ZUP» se traduit notamment dans les relations courtoises mais distantes qu'ils entretiennent avec ses habitants. M. et Mme Chabot sont là " parce que c'est pratique pour le boulot " du mari. Ils ne sauraient s'encombrer d'une sociabilité inappropriée. Cette posture alimente un système complexe de braconnages dans les découpages spatiaux administratifs et les temporalités urbaines. Ainsi, à la fin de mon enquête s'est posé le problème de l'inscription de l'enfant à l'école. Soucieux de lui fournir un environnement scolaire favorable, ses parents n'ont pas souhaité l'inscrire à l'école du quartier. Fréquentée par « les gens de la ZUP », elle leur apparaissait comme un milieu peu adapté à l'éducation de leur fils. Le jeune couple a alors dû affronter les logiques de la carte scolaire. Quelques « relations » à la mairie allaient être mobilisées pour échapper à l'assignation des pratiques aux découpages administratifs. En dernier recours, l'école privée pourrait s'imposer comme une alternative. M. et Mme Chabot se sont alors livrés à une construction spatio-temporelle complexe. Temps contraint des transports quotidiens, horaires actuels et prévisibles de travail et contrôle des lieux de la socialisation qui s'imposent à leurs pratiques quotidiennes venaient s'inscrire dans un système d'ambitions sociales. Loin d'aller de soi, cette construction spatiotemporelle se présente comme un double braconnage : d'une part dans les découpages administratifs, d'autre part dans les temporalités qui pèsent sur le quotidien. M. et Mme Chabot projettent et organisent un usage quotidien de l'espace. Ils découpent, tranchent, inscrivent dans le temps et combinent rythmes de la ville et rythmes du foyer. Ils construisent un bloc d'espace-temps cohérent avec leur champ des possibles.

\section{Délimiter les territoires du quotidien}

17 Les territoires du quotidien prennent des formes personnelles et familiales, multiples et changeantes. Enchevêtrés, ils présentent des limites brouillées. Pour faire frontière, les habitants se livrent à une activité productrice matérielle et symbolique. Chaque fois, 
celle-ci apparaît en des lieux investis d'une forte capacité à signifier un ordre social et culturel. Elle est construite comme une séparation structurante qui exprime ou révèle l'exercice d'un pouvoir. Mais les territoires du quotidien mettent à jour des réalités frontalières qui en renouvellent les expressions conventionnelles. Trois manières de faire frontière peuvent être ici repérées ${ }^{4}$ qui se distinguent par leurs formes et leurs fonctions.

\section{Faire frontière réticulaire}

Miloud a 19 ans. Il est "des barres ». Il habite avec ses parents et ses cinq frères et sœurs dans ces bâtiments de quatre étages long d'une centaine de mètres. Au moment de l'enquête, Miloud est sans emploi. Cuisinier de formation, il n'a pas trouvé de travail depuis sa sortie du lycée professionnel. Une partie de ses journées et de ses soirées se déroule, au pied des tours où il sait pouvoir retrouver un petit groupe de jeunes du quartier. Il reste parfois jusqu'à une heure avancée de la nuit. «De toute façon, constate-t-il, y a que ça à faire. » Une plongée attentive dans ses pratiques quotidiennes laisse apparaître une appropriation de l'espace sensiblement plus complexe et étendue. Miloud est très mobile. Pour "traîner en ville ", "chercher du taf »... il trace dans l'agglomération des chemins diversifiés. En s'évadant du quartier pour échapper à «la galère ", il investit et relie d'autres lieux, active d'autres liens et combat un pesant sentiment d'assignation. Alors, parfois seul, souvent en bande, Miloud entretient dans la ville un réseau de lieux et un réseau de liens. Pour lui ce qui fait territoire c'est ce qui fait groupe. "Quand tu vas traîner le samedi à Continent sur le coup des 14 heures, obligé tu vois du monde. Je suis trop connu là-bas. » Il y a aussi quelques bars du centre de Tours le soir, la maison de quartier les après-midi de semaine, l'un des porches des tours à des heures précises de la soirée. Son appréhension de la ville laisse apparaître un réseau de microblocs d'espace-temps que Miloud entretient avec soin. Ces blocs constituent autant de possibilités de rendez-vous reliés par des flux de communication. «On va en ville souvent. Enfin pas n'importe où. Y a des coins qui sont pas bons pour les Arabes, comme vers la caserne. C'est trop plein de racistes. Pis y a d'autres coins, quand tu dis que t'es de la Rabaterie, tu te fais latter comme vers le Sanit ${ }^{5}$." Les microblocs d'espace-temps apparaissent séparés entre eux plus que par une simple limite. Se sont véritablement des formes frontalières qui s'expriment dans le sens ou se sont chaque fois des ruptures d'exercices de pouvoir qui sont marquées et signifiées. Miloud et sa bande mettent à jour des formes frontalières inhabituelles. Ces formes sont caractérisées par la discontinuité. Elles ne prennent pas la forme de lignes. Elles consistent en une multitude de points de contrôles qui se multiplient au sein d'espace réticulaire. Elles se localisent et s'imbriquent dans des réseaux tracés pour relier les lieux du quotidien.

\section{Faire frontière sociale}

Les frontières les plus sélectives peuvent être au plus près de l'intime. Ainsi, en est-il de la porte d'entrée de la famille Choquet. De l'extérieur, aucun signe distinctif n'est visible. Le nom n'est pas inscrit sur la sonnette. Le paillasson tout simple est celui qui avait été laissé par le locataire précédent. Cette porte très ordinaire agit pourtant comme une frontière à la redoutable efficacité qui puise ses raisons et ses ressources dans la trajectoire sociale et résidentielle des occupants. Installés là en attendant 
d'accéder au rêve pavillonnaire présenté comme imminent, M. et Mme Choquet opèrent une vigoureuse mise à distance des "gens d'ici » qu'ils ne pensent pas susceptibles de quitter un jour le quartier. Cette posture justifie le renforcement de la frontière appartement/palier et la mise en œuvre d'un contrôle strict des modalités de franchissement. Le seuil apparaît puissamment sélectif. Plus on est voisin et moins on a de chances d'entrer. «Les voisins, raconte Mme Choquet, c'est bonjour-bonsoir [...]. Ça nous viendrait pas à l'idée de les inviter." La volonté d'accéder à un mode de vie supérieur à celui des "gens d'ici » justifie et alimente le refus de la confrontation. « On les voit pas. On n'est pas fâché, loin de là mais on n'irait pas inviter tous les voisins [...] Ils n'ont pas besoin de savoir comment c'est chez nous. Je dis pas qu'ils sont tous malhonnêtes les gens ici. Mais, il y a des trucs [elle désigne la chaîne hifi], c'est pas la peine d'exciter la jalousie. Alors en général, ils restent sur la porte. » Cette porte sépare deux mondes. Elle opère une distinction entre «l'extérieur» et le logement qui constitue un champ symbolique spécifique assurant la reproduction et l'entretien de l'identité familiale.

21 Pour matérialiser cette frontière, M. et Mme Choquet ont constitué une entrée. Comme dans tous les appartements du même type, l'espace séparant le seuil de la pièce principale est très exigu. Pourtant, comme beaucoup de voisins, les Choquet ont voulu faire une entrée. Celle-ci est construite selon des modalités expérimentées dans des emménagements antérieurs du couple ou de ses membres encore célibataires. $\mathrm{Ni}$ ostentation du foyer, ni objet de négligence, elle rassemble les éléments signifiant du passage. Un paillasson intérieur acheté à Casa, un meuble à chaussures récupéré chez les parents de monsieur, un portemanteau perroquet acheté par le jeune couple pour le studio de Tours, aujourd'hui trop grand pour son nouvel emplacement... L'ameublement de l'entrée signifie la frontière et raconte l'histoire d'une famille à ses propres membres. La construction de cette entrée est le fruit d'une opération synthétique particulière. Elle résulte d'un acte d'imagination qui met en correspondance une détermination spatiale et une détermination conceptuelle. L'imagination à l'œuvre ici est telle qu'elle avait été décrite par Gilles Deleuze : une faculté par laquelle l'individu détermine un espace de manière conforme à un concept mais qui ne découle pas du concept. L'imagination est productrice et non reproductrice. Une fois constituée, l'entrée prend toute sa dimension frontalière, comparable dans sa configuration aux frontières sociales que Georg Simmel définissait comme une limite marquant une différence de degré de participation à la société entre différents groupes. La frontière sociale n'est pas pour autant une simple métaphore spatiale. Elle produit de bien réelles limites spatiales perçues et vécues par les intéressés.

\section{Faire frontière gestionnaire}

Propreté des halls, chiens dans les aires de jeu... parfois, l'entretien des parties communes pose problème. Parfois, « les HLM » ne jouent pas pleinement le rôle que les habitants en attendent. Alors, des acteurs alternatifs viennent assurer des encadrements de substitution. Associations de quartier, réseaux informels de voisins... s'approprient des espaces qui se trouvent alors soumis à un contrôle spécifique du groupe concerné. De nouveaux territoires s'esquissent. De nouvelles frontières apparaissent. 
23 À proximité des tours de l'Aubrière, une aire de jeux a été aménagée avec toboggans colorés et pingouins sur ressorts. Régulièrement, en matinée ou en début de soirée, les chiens viennent volontiers souiller les revêtements de sol, parfois surveillés à distance par quelques maîtres désinvoltes. Le soir, quelques adolescents s'y retrouvent bruyamment, y fument, y boivent parfois. Ils détournent les usages et les horaires du lieu, se l'approprient de manière non conforme. Alors, spontanément, un groupe de jeunes mères de familles s'est créé. Le rendez-vous quotidien de la sortie d'école a permis la constitution d'un collectif gestionnaire, informel mais bien réel. La composition de celui-ci fut d'autant plus incertaine que l'hétérogénéité sociale du grand ensemble limite profondément l'établissement d'un système de normes partagées. Dans ce contexte, les initiatives collectives peinent à trouver leur légitimité. Pourtant, mobilisant un ensemble de moyens techniques et symboliques, quelques jeunes mères ont décidé de faire frontière, d'affirmer leur contrôle sur le lieu et d'en interdire l'accès à ceux qui en faisaient un usage non conforme. Affichettes, signalement aux autorités compétentes leur sont apparus comme autant d'outils disponibles.

24 Ce groupe de jeunes mères se constitue et se perpétue dans et par cette activité de défense territoriale. La frontière gestionnaire ainsi créée reste d'une efficacité circonscrite au champ du symbolique. Elle n'en est pas moins capable de revêtir une grande signification pour le groupe concerné. Elle renvoie à une communauté pertinente d'action collective cristallisant des projets personnels comprenant une dimension altruiste (participer à un maintien de l'ordre public) et une dimension égoïste (adhérer à un collectif de pairs dans une société locale hétérogène et profondément marquée par le repli domestique). Mais elle demeure limitée dans le temps. À la fin de l'enquête, les affichettes avaient disparu.

\section{Entretenir les territoires du quotidien}

25 Le propre de la démarche ethnologique est d'installer l'observation dans le temps long et d'impliquer le chercheur dans les pratiques observées. Acteur et témoin, il voit à l'œuvre un registre particulier de logiques d'action. Ni savantes, ni techniques, elles relèvent de l'ordinaire. Se sont les logiques du familier, ces logiques implicites des comportements qui "vont de soi " et renvoient à des opérations matérielles et symboliques que l'on reproduit sans réinterroger mais que Michèle de La Pradelle invitait à prendre au sérieux. Leur mise en œuvre permet d'explorer deux caractères majeurs des territoires du quotidien : les créations de familiarité et de sécurité.

\section{Rendre familier}

Après sa "journée de boulot ", M. Croizer rentre chez lui. Parmi les espaces de son quotidien, il en est un dont il s'emploie à faire territoire. C'est son «chez soi ». L'exemple de M. Croizer illustre le principe largement répandu dans le grand ensemble du repli domestique et de la survalorisation du logement par rapport aux parties communes. Chez lui, M. Croizer se repose du «n'importe quoi» qui à ses yeux caractérise «la ZUP» et le collège du quartier dans lequel il travaille. "Dans [ses] meubles ", M. Croizer se ressource. Il est dans un espace dont il domine l'organisation et qui lui apparaît paisible et ordonné. Cet ordre intérieur agit comme un repère garde- 
fou du soi. Il contribue, pour reprendre une expression de Jean-Claude Kaufmann, à construire et jalonner une identité dispersée, contradictoire et mouvante (Kaufmann, 1988). Le chez-soi se met au service d'un processus de de-altération. « On ferme la porte, on est chez soi. » C'est un autre univers qui commence, débarrassé de l'emprise de l'autre. La dimension temporelle participe de cette création de familiarité. Les territoires du quotidien sont aussi affaire de rythmes. Ils s'organisent et se perpétuent dans la régularité et la répétition. Ainsi, une fois chez lui, quelques manipulations routinières d'objets permettent à $\mathrm{M}$. Croizer de «ne plus [se] sentir au boulot». Quelques microrituels quotidiens jalonnent une domesticité réparatrice. La consultation du répondeur, l'ouverture du courrier... constituent un univers de gestes et de sons familiers qui participent à la fabrication du «chez-soi ». La routine, en tant qu'habitude d'agir toujours de la même manière de façon mécanique et irréfléchie entre dans le procédé de fabrication des territoires du quotidien. Elle permet de développer une habileté singulière acquise par l'expérience et la pratique. Par elle, l'apparentement et la familiarité des territoires du quotidien prennent corps et se réalisent concrètement.

Ainsi la création de familiarité permet d'envisager la fabrication des territoires du quotidien comme un processus permanent d'entretien et de maîtrise de blocs d'espacetemps non seulement débarrassés de l'emprise de l'autre mais également investis d'une capacité de de-altération. Plus cette capacité de-altérante se crée, plus les espaces du quotidien se font territoires. Les frontières viennent alors verrouiller, stabiliser pour un temps cette création qui sera validée par la pratique et façonnée par la routine.

\section{Construire des espaces-temps sécurisés}

Au début de l'enquête, Nadia a 20 ans. Elle habite depuis l'enfance un appartement de la Rabaterie avec ses parents et ses frères et sœurs. Elle travaille à l'autre extrémité de l'agglomération, au restaurant Quick de Chambray. Lors des entretiens, Nadia justifie souvent le choix et l'organisation de ses pratiques quotidiennes par le fait qu'elle est « une beurette ». Elle mobilise cette appartenance ethnique, de genre et de génération pour configurer sa construction d'espace-temps du quotidien. Celle-ci embrasse la métropole tourangelle tout entière. Chaque jour, Nadia se rend en bus à son travail. Régulièrement, elle fréquente les équipements de quartier (la maison de quartier, le centre commercial...). Dès qu'elle le peut, elle va « dans Tours faire les boutiques avec les copines». Le territoire du quotidien se tisse dans des situations sociospatiales qui lui permettent une effervescence groupale avec le faisceau d'appartenances qu'elle revendique. Elle se livre à une construction territoriale réticulaire comparable à celles qui ont pu être décrites précédemment. Mais, l'exemple de Nadia permet aussi d'éclairer un volet important des territoires du quotidien: c'est la dimension sécuritaire. À ses yeux, il ne peut y avoir de blocs d'espace-temps délimité et familier qui ne puisse garantir sa sécurité. La fabrication du territoire du quotidien est indissociable d'une construction sécuritaire. Celle-ci se fait dans un jeu sur l'espace et le temps. Ainsi, Nadia évite-t-elle soigneusement les «endroits qui craignent». Si elle "traîne sur le quartier » durant la journée, elle s'interdit d'y rester le soir. Certains retours du travail la font se dérouter. Parfois, il lui faut traverser des espaces qui, bien que connus, cessent de lui paraître sécurisés. La sécurité des lieux est aussi affaire de moment. Pour agir en conséquence, Nadia mobilise sa connaissance de la ville et de ses 
acteurs. Elle connaît les endroits éclairés et animés. Elle sait où et quand elle sera reconnue et identifiée. Elle connait les rythmes de la ville et mobilise des capitaux sociaux, et économiques pour mesurer les risques et construire ses blocs d'espacetemps en conséquence. Jouant sur les horaires et les trajets, Nadia pose des jalons, construit des refuges.

Espace de connaissance et de reconnaissance des personnes, des lieux et des objets, ce territoire sécurisé est aussi celui de l'impossible anonymat. Ce qu'il offre de sécurité, il le fait perdre en liberté. "Trop connue » dans son quartier, Nadia se sent parfois trop surveillée. L'aspect clos et volontiers étouffant lui apparait comme le pendant négatif de la sécurisation. Alors, elle quitte régulièrement les sentiers battus pour fréquenter des lieux en anonyme. Ainsi, de la même manière qu'ils sont faits d'extérieurs et d'intérieurs, les territoires du quotidien semblent pour se réaliser, nécessiter la coprésence ou au moins la co-possibilité de clos et d'ouvert, de reconnaissance et d'anonymat, de sécurité et de liberté.

Dans les appartements ou les parties communes, dans la ville et ses interstices, des rêves pavillonnaires, des préoccupations ménagères... s'expriment et s'impriment. Saisissant la matérialité et la temporalité des lieux, ils participent à la production de territoires du quotidien singuliers. Les pratiques routinières s'inscrivent dans des ensembles disparates d'espaces et de lieux que chacun relie ou disjoint en faisant appel à ses habitudes et ses fantaisies, à ses souvenirs et ses projets. Bricolé, hétérogène, l'assemblage formé est volontiers discontinu, animé par des rythmes et des horaires multiples.

Réflexion en cours, cette revisite de matériaux d'enquête n'avait d'autres ambitions que d'explorer la pertinence d'une approche de la question "à hauteur d'habitant " en terme de fabrication. À l'évidence, il est possible de rechercher la cohérence dans cet ordre dispersé en pratiquant une anthropologie des singularités subjectives, attentive aux actes ordinaires et aux transformations minuscules auxquelles chacun se livre pour constituer des blocs d'espace-temps. Ceux-ci structurent les situations sociospatiales du quotidien en articulant les propriétés intrinsèques des espaces avec des systèmes de dispositions sociales, économiques et culturelles. Il est parfois intéressant, pour reprendre une formule de Claude Javeau, de prendre le futile au sérieux. Alors, il devient envisageable d'accéder aux logiques d'inventions contextuelles qui font territoires du quotidien. La démarche proposée ici ne permet pas de cerner de manière exhaustive les territoires du quotidien mais de comprendre un peu des ressorts de leur genèse et de leurs transformations.

\section{BIBLIOGRAPHIE}

Alphandéry P., Bergues M., 2004, « Territoires en questions. Pratiques des lieux, usages d'un mot », Ethnologie française, $n^{\circ} 1$, p. 5-12. 
Bergues M., 1998, Les relations aux lieux en pays Beaumontois, Mission du Patrimoine Ethnologique/ Communauté de communes du pays Beaumontois.

Bonetti M., 1994, Habiter. Le bricolage imaginaire de l'espace, Paris, Desclée de Brouwer.

Bromberger C., Ravis-Giordanni G., 1976, «Introduction : Espace donné, espace produit ; esquisse d'une approche ethnologique du concept d'espace ", in Balfet H. et al. (dir.), Pratiques et représentations de l'espace dans les communautés méditerranéennes, Paris, Éditions du CNRS, p. 13-25.

Bromberger C., Meyer M., 2003, «Cultures régionales en débat », Ethnologie française, $\mathrm{n}^{\circ} 3$, p. 357-361.

Certeau M. (de), 1980, L'invention du quotidien I. Arts de faire, Paris, Gallimard.

Chamboredon J.-C., Lemaire M., 1970, « Proximité spatiale, distances sociales. Les grands ensembles et leur peuplement ", Revue française de sociologie, vol. XI, p. 3-33.

Javeau C., 1998, Prendre le futile au sérieux. Microsociologie des rituels de la vie courante, Paris, Cerf.

Kaufmann J.-C., 1988, La chaleur du foyer. Analyse du repli domestique, Paris, Méridien Klincksieck.

La Pradelle M. (de), 1996, Les vendredis de Carpentras. Faire son marché en Provence ou ailleurs, Paris, Fayard.

Le Berre M., 1995, « Territoires », in Bailly A., Ferras R., Pumain D., (dir.), Encyclopédie de géographie, Paris, Economica, p. 617-638.

Rémy J., 1996, « Mobilités et ancrages : vers une autre définition de la ville », in Hirschhorn M., Berthelot J.-M. (dir.), Mobilités et ancrages. Vers un nouveau mode de spatialisation?, Paris, L'Harmattan.

Sencébé Y., Hilal M., 2003, « Espaces et territoires périurbains : des logiques de déplacement aux logiques d'ancrage », colloque Travail et territoires, Confrontation d'approches disciplinaires en économie, histoire et sociologie, MSH de Bourgogne, Dijon, 13 au 13 mars.

Simmel G., 1999, Sociologie. Études sur les formes de socialisation, Paris, PUF.

\section{NOTES}

1. C'est le terme employé par la presse locale pour qualifier les événements du printemps 1996 et de l'automne 2005.

2. Voir D. La Mache : L'art d'habiter un grand ensemble HLM, Paris, L'Harmattan, 2006.

3. C'est-à-dire à délimiter des ensembles spatiaux appropriés dont les individus se donnent la liberté de disposer chaque jour, un espace qui relève d'un modèle de découpage, d'usage et de contrôle propre à l'individu considéré et pourvu d'un champ symbolique spécifique, garant d'identité.

4. Par bien des aspects, ces manières rappellent les configurations décrites par C. ArbaretSchulz, A. Beyer, J.-L. Permay, B. Reitel, C. Selimanovski, C. Sohn et P. Zander in « La frontière, un objet spatial en mutation ", EspaceTemps.net, 29 octobre 2004.

5. Un autre quartier d'habitat social voisin. 


\section{RÉSUMÉS}

Cet article repose sur une étude ethnologique menée auprès des habitants d'un grand ensemble HLM de la banlieue tourangelle. Il s'agit de mettre à jour les inventions contextuelles et les arts de faire par lesquels, dans la répétitivité de leurs pratiques quotidiennes, ces habitants délimitent, administrent, transforment matériellement et symboliquement des espaces et des lieux, en domptent les temporalités propres pour contribuer à faire territoire et entretenir, transformer ou préserver des identités personnelles ou familiales. Nous aborderons la manière dont se fabriquent les territoires du quotidien. Ensuite, sera posée la question de leur délimitation. Pour finir, nous traiterons la manière dont les territoires du quotidien sont gérés et entretenus par leurs auteurs.

This article is based on an ethnological study conducted among the inhabitants of a low-income housing scheme in the suburbs of Tours. The issue is to reveal contextual inventions and manners of doing things by the inhabitants who, through their repeated daily practices, delimit, manage, transform spaces and places - either materially or symbolically - by mastering their own temporalities to contribute to make territory and maintain, transform or preserve personal or family identities. We shall look into how everyday life territories are built up. We shall then address the issue of their delimitation. Lastly, we shall look at the way everyday life territories are managed and maintained by their authors.

\section{INDEX}

Mots-clés : habitat, quotidien, espace urbain, territoire, identités, espace périurbain Index géographique : France

\section{AUTEUR}

\section{DENIS LA MACHE}

Anthropologue, responsable de formation au CREPS Poitou-Charentes

Ladyss (Laboratoire dynamiques sociales et recomposition des espaces),

CNRS / univ. Paris 1

2 rue Valette,

75005 - Paris

denislamache@yahoo.fr 